\title{
SPECIFICATION OF MULTIPOLE TOLERANCES FOR THE APS QUADRUPOLE MAGNET
}

This note will address a proposed method for specifying the multipole tolerance for the design and production of APS quadrupole magnets. The tolerances for the multipole components for the quadrupole magnets will be set to that level which reduces the dynamic aperture by about $10-15 \%$ from the Ideal machine dynamic aperture (as specified in CDR-87). This level may appear rather stringent, especially compared to the $50-60 \%$ reduction resulting from quad placement errors. However, when all tolerances are taken together, the residual dynamic aperture would be prohibitively small and commissioning would be difficult if these tolerances were at twice this level. The dynamic aperture was determined using the numerical tracking program RACETRACK. (1)

The multipole tolerances are defined by the expansion of the magnetic fleld given in Section II.4.2 of CDR-87. For quadrupoles $a_{0}, b_{0} \equiv 0$ (quadrupole placement tolerances handle these dipole terms) and $b_{1} \equiv 1.0 \mathrm{~cm}^{-1}$ with $\left.\frac{d B}{d x}\right|_{y=0}=B_{0} b_{1}$ and $\left.\frac{d B}{d y}\right|_{x=0}=-B_{0} a_{1}$. Each of the 17 coefficients will have a tolerance value for the average value $\left(\bar{b}_{n}\right)$ of that coefficient over the ensemble of magnets ( 400 required) and for the spread or standard deviation $\left(\delta b_{n}\right)$ which accounts for magnet-to-magnet varlations. In the tracking program, the $\left(\delta b_{n}\right)$ is taken to specify the rms spread of the magnet-to-magnet variations. The actual value for each $b_{n}$ is assumed to be a random variable with a Gaussian distribution of mean $\bar{b}_{n}$ and standard deviation $\delta b_{n}$ : No reordering or shuffling of magnets was used in computing the dynamic aperture, although this will be used to position magnets in the actual accelerator. The calculated dynamic aperture is the average of that obtained by generating eleven machines with different random sequences of magnet coefficients. nietaraimION OF THIS DOCUMENT IS UNLIMITED R 


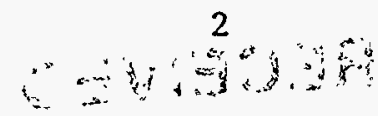

With 34 tolerances to be spectified, the computational problem is undefined unless some relative scalling of the coefficients can be chosen. Although calculational techniques may be useful in setting levels for the allowed coefficients, the $\delta b_{n}$ and non-allowed coefflcients are difficult to compute in a way which will match the construction imperfections. In this case, we will use the relative magnitudes of these 34 tolerances taken from measurements of magnets built for two existing storage rings. Although different in design details from the APS quads, these two sets of magnets represent a very high-quality magnet (PEP insertion quad) and a lower-quality but more reasonable mass-produced magnet (FNAL pbar source quads). The high-quality multipole coefficients we used were obtained from the summary of the PEP insertion quadrupoles quoted in the LBL 1-2 GeV synchrotron Light Source $C D R(2)$ but scaled to the same $4-\mathrm{cm}$ aperture as the APS quads (see Appendix). The lower-quality multipole coefficients were obtained from measurements $(3)$ of the FNAL SQA 500 series quadrupoles. The actual data for these measured integrated coefficients are shown in Table I, together with calculated ${ }^{(4)}$ values for the APS quads with a non-optimized design for the quadrupole ends. The scaled $a_{n}$ and $b_{n}$ 's assumed for the APS quadrupoles, with a 4-cm aperture radius, are given in Table II.

Figure 1 shows the effect on the dynamic aperture using the scaled coefficients from the PEP insertion quadrupoles. The data points and error bars represent the average and rms spread of the dynamic aperture for the 11 different sets of randomly distributed coefficients. Very little change from the ideal dynamic aperture is observed.

Figure 2 shows the effects of the more realistic FNAL quadrupole coefficients. The reduction of the dynamic aperture is about $10-15 \%$ and therefore represents the tolerance of the harmonic coefficients necessary for 


\section{DISCLAIMER}

This report was prepared as an account of work sponsored by an agency of the United States Government. Neither the United States Government nor any agency thereof, nor any of their employees, makes any warranty, express or implied, or assumes any legal liability or responsibility for the accuracy, completeness, or usefulness of any information, apparatus, product, or process disclosed, or represents that its use would not infringe privately owned rights. Reference herein to any specific commercial product, process, or service by trade name, trademark, manufacturer, or otherwise does not necessarily constitute or imply its endorsement, recommendation, or favoring by the United States Government or any agency thereof. The views and opinions of authors expressed herein do not necessarily state or reflect those of the United States Government or any agency thereof. 


\section{DISCLAIMER}

Portions of this document may be illegible in electronic image products. Images are produced from the best available original document. 
Table I

Summary of FNAL SQA500 and PEP* Insertion Quadrupole Harmonic Measurements

\begin{tabular}{|c|c|c|c|}
\hline $\begin{array}{c}\text { Harmon } \\
n\end{array}$ & $\begin{array}{l}\mathrm{FNAU}\left(\mathrm{r}_{0}=4.306\right. \\
\mathrm{B}(\mathrm{n}) / \mathrm{B}(1) \\
\times 10^{-4}\end{array}$ & $\begin{array}{c}\mathrm{cm}, 1=4.445 \mathrm{~cm}) \\
\mathrm{A}(\mathrm{n}) / \mathrm{B}(1) \\
\times 10^{-4}\end{array}$ & $\begin{array}{c}\operatorname{PEP}\left(r_{0}=a=8 \mathrm{~cm}\right) \\
B(\underline{n}) / B(I) \\
\times 10^{-4}\end{array}$ \\
\hline 0 & $29.4 \pm 78.5$ & $-4.71 \pm 43.7$ & $-\infty$ \\
\hline 1 & $0.996 \pm .022 \times 10^{4}$ & $17.35 \pm 61.3$ & 1.0 \\
\hline 2 & $-0.77 \pm 2.08$ & $-1.94 \pm 4.35$ & $0.0 \pm 0.0128$ \\
\hline 3 & $-4.15 \pm 1.70$ & $-0.35 \pm 0.49$ & $0.0 \pm 0.853$ \\
\hline 4 & $-.164 \pm 0.36$ & $0.32 \pm 0.42$ & $0.0 \pm 0.000$ \\
\hline 5 & $-3.15 \pm 0.77$ & $0.05 \pm 0.22$ & $0.61 \pm 0.41$ \\
\hline 9 & $3.48 \pm 0.15$ & $-0.07 \pm 0.14$ & $-0.30 \pm 0.15$ \\
\hline
\end{tabular}

* Obtained from the ALS Conceptial Design Report, LBL (1986).

Table II

Scaled Harmonic Coefficients Used for Model of the APS Quadrupole Harmonic Coefficient Tolerances*

\begin{tabular}{cccc}
\hline & \multicolumn{3}{c}{$\# 1$} \\
$n$ & $b_{n} \times\left[\mathrm{cm}^{-(n)}\right]$, & $b_{n}\left[\mathrm{~cm}^{-(n)}\right]$ & $a_{n}\left[\mathrm{~cm}^{-(n)}\right]$ \\
\hline 1 & 1.0 & 1.0 & $17.4 \pm 61.3 \times 10^{-4}$ \\
2 & $0.0 \pm 5.0 \times 10^{-6}$ & $-1.9 \pm 5.4 \times 10^{-5}$ & $-5.0 \pm 11.2 \times 10^{-5}$ \\
3 & $0.0 \pm 5.3 \times 10^{-6}$ & $-2.8 \pm 1.1 \times 10^{-5}$ & $-2.3 \pm 3.3 \times 10^{-6}$ \\
4 & $0.0 \pm 0.0$ & $-2.8 \pm 6.2 \times 10^{-7}$ & $5.5 \pm 7.2 \times 10^{-7}$ \\
5 & $0.24 \pm 0.16 \times 10^{-6}$ & $-1.4 \pm 0.3 \times 10^{-6}$ & $0.2 \pm 1.0 \times 10^{-7}$ \\
9 & $-0.45 \pm 0.25 \times 10^{-9}$ & $6.9 \pm 0.3 \times 10^{-9}$ & $-1.4 \pm 2.8 \times 10^{-10}$ \\
\hline
\end{tabular}

* Scaled assuming the normalized coefficients are the same as for the measured magnets. 
APS quads. However, the average value tolerances can be increased by 2-3 times without significantly reducing the dynamic aperture. The rms value of the harmonic coefficients dominates the effect on the dynamic aperture, but this could probably be improved by repositioning of the quads to suppress the dominant resonance terms. The algorithm for reordering will not be considered here, but will be discussed in a future note. Figure 3 shows the impact on the dynamic aperture from increasing the random coefficients by factors of 2 and 5 times the FNAL coefficients.

In the $1987 \mathrm{CDR}$, the tolerance on the harmonic coefficients was calculated in the same manner as described here, but only the duodecapole term $\left(b_{5}\right)$ was considered (all other $b_{n}, a_{n}=0$ ). In that case, the average value was limited to $\left|\bar{b}_{5}\right|<5 \times 10^{-6} \mathrm{~cm}^{-4}$ taken to be positive or negative for all quadrupoles, regardless of the sign of the gradient. However, this tolerance can be increased by a factor of 20 or more if the sign of $\bar{b}_{5}$ depends on the sign of the gradient (i.e., magnitude of gradient increases or decreases for all quads). The tolerance on the spread was $\delta b_{5} \leqslant 2.5 \times 10^{-6} \mathrm{~cm}^{-4}$ or a factor of 7.5 times the FNAL value specified here. This demonstrates the dependence of the tolerance specification on the number of coefficients considered and their relative scaling.

The APS quadrupole design is quite different from that of the PEP or FNAL magnets. Consequently, the multipole coefficients have been calculated(5) (in 2-D) in order to estimate the size of the average value $\left(\bar{b}_{n}\right)$ that might result from production.* Table III shows these values compared to the scaled average values of the FNAL quadrupoles. All coefficients are quite similar in magnitude for these two sets of data, except for the $b_{4}$ term, which is almost programs with small changes in radial aperture. 
Table III

Calculated Multipole Coefficients for APS Quadrupoles

Compared to the Scaled FNAL Average Values.

\begin{tabular}{lllll}
\hline & \multicolumn{2}{c}{$\mathrm{b}_{\mathrm{n}}\left(\times 10^{-6} \mathrm{~cm}^{-\mathrm{n}}\right)$} & $\begin{array}{c}a_{\mathrm{n}}\left(\times 10^{-6} \mathrm{~cm}^{-\mathrm{n}}\right) \\
\text { APS }\end{array}$ & FNAL \\
\hline 2 & APS & FNAL & & \\
\hline 3 & 14 & -19 & 75 & -50 \\
4 & 8.0 & -28 & 0.0 & -2.3 \\
5 & 2.7 & -0.28 & 0.0 & 0.55 \\
6 & 6.3 & -1.4 & 0.0 & 0.02 \\
7 & 0.4 & 0.0 & 0.0 & 0.0 \\
8 & 0.14 & 0.0 & 0.0 & 0.0 \\
9 & 0.045 & 0.0 & 0.0 & 0.0 \\
\hline
\end{tabular}

ten times the FNAL average value. The dynamic aperture has been calculated using the calculated $a_{n}, b_{n}$ for the APS design for $\bar{a}_{n}, \bar{b}_{n}$ and the FNAL $\delta b_{n}, \delta a_{n}$. The resulting dynamic data differ only in the rms spread of dynamic aperture being slightly smaller than that shown in Fig. 2. Increasing the average values by factors of 2 and 5 times shows a small reduction of the dynamic aperture, as presented in Fig. 4. This supports the idea that it is the spread of the multipole coefficients which dominates the reduction of the dynamic aperture.

In conclusion, the dynamic aperture reduction of $10-15 \%$ is expected if the average value and spread of the multipole coefficients in the APS quads are simflar to those measured in the FNAL quadrupoles. This sets the tolerance Ievel for the APS quadrupole according to the CDR-87 standard. The 
dominant terms, in terms of their impact on the dynamic aperture, are the $\delta b_{n}$, $\delta a_{n}$. Larger values for these terms may be acceptable but could limit the - aperture with quad alignment errors, unless an effective magnet positioning algorithm is developed. Larger average values, up to two or three times the FNAL $\vec{b}_{n}, \vec{a}_{n}$, may be acceptable if the $\delta b_{n}, \delta a_{n}$ don't also increase.

REFERENCES

1. A. Wrulich, "RACETRACK, A Computer Code for the Simulation of Nonlinear Particle Motion in Accelerators," DESY 84-026 (1984).

2. A. Avery et al., "1-2 GeV Synchrotron Light Source," LBL PUB-5172 Rev July 1986 and IEEE NS-26, p. 4033 (1979).

3. B. Brown, "Summary Sheets for SQA500 Measured Harmonic Components," private comm. (1988).

4. R. Lari, Argonne National Laboratory, private communication (March 1988).

5. S. Kim, Argonne National Laboratory, private communication (August 1988). 
APPENDIX

The magnetic-field calculation programs and measurements with coils yield harmonic field components specified within a given coil radius $\left(r_{0}\right)$ relative to that of the first harmonic. For quadrupoles these are defined as

$$
\left.\frac{B(n)}{B(1)}\right|_{z=r_{0}}=\frac{\int B_{0} b_{n} r_{0}^{n} d l}{\int B_{0} b_{1} r_{0} d l}=\frac{b_{n}}{b_{1}} r_{0}^{n-1}
$$

and

$$
\left.\frac{A(n)}{B(1)}\right|_{z=r_{0}}=\frac{\int B_{0} a_{n} r_{0}^{n} d l}{\int B_{0} b_{1} r_{0} d l}=\frac{a_{n}}{b_{1}} r_{0}^{n-1}
$$

where the $b_{n}$ and $a_{n}$ represent the effective coefficients over the entire quad. In order to compare magnets with differing aperture radius $a$, the $a_{n}$ and $b_{n}$ will be scaled (best estimate for magnets with equal care in designing the pole tips ${ }^{(5)}$ ) as the percentage of the aperture that the measurement radius $r_{0}$ represents. The normalized harmonic coefficients are defined as

$$
B_{y}+i B_{x}=B_{0} \sum_{n=2}\left(b_{n}^{a}+i a_{n}^{a}\right)\left(\frac{z}{a}\right)^{n}
$$

The $a_{n}^{a}$ and $b_{n}^{a}$ coefficients will allow for a more straightforward comparison of the harmonic strengths of different magnets with different apertures. The relation between the coefficients used by RACETRACK are given by

$$
a_{n}, b_{n}=\frac{a_{n}^{a}}{(a)^{n}}, \frac{b_{n}^{a}}{(a)^{n}}
$$


The values used in this dynamic aperture study are related to the measured values for a magnet with aperture radius $a$, by the relations

$$
a_{n}(\text { APS }), b_{n}(\text { APS })=\left(\frac{a}{a_{\text {APS }}}\right)^{n} \times a_{n} \text { (measured), } b_{n} \text { (measured) }
$$

with $a_{A P S}=4 \mathrm{~cm}$. This assumes that $a_{n}^{a}, b_{n}^{a}$ are equal for the two magnets. A final specification of the harmonic coefficients used by the programs DIMAT and MAD are the normalized gradient coefficients (for $a_{\mathfrak{n}}=0$ )

$$
\bar{B}=B_{\rho} \sum_{m=0} \frac{K_{m} r^{m}}{m !}
$$

and

or

$$
\frac{B(n)}{B(1)}=\frac{K_{n} \cdot r_{0}^{n-1}}{K_{1}(n) !}=\frac{b_{n}^{a}}{b_{1}^{a}}\left(\frac{r}{a}\right)^{n-1}
$$

$$
\frac{b_{n}^{a}}{b_{1}^{a}}=\frac{k_{n}}{k_{1}} \frac{(a)^{n-1}}{(n) !}
$$

The measured values of the PEP insertion quadrupoles have been summarized by LBL in their CDR-86. (2) We have scaled these values for $a=r_{0}=8 \mathrm{~cm}$ to the APSdesigned quadrupole magnets with $a_{A P S}=4 \mathrm{~cm}$, as shown in Table II. The FNAL quadrupoles were measured with a coil of $r_{0}=4.3 \mathrm{~cm}$ and have an aperture of $a=4.45 \mathrm{~cm}$. We have scaled their measured data to the APS aperture, as presented in Table II. No reduction of random coefficients has been included for measurement resolution of the harmonic coefficients. For some coefficients $b_{4}, a_{4}$, and $a_{5}$, the entire systematic and random coefficients could be due to the measurement resolution and finite statistics. 


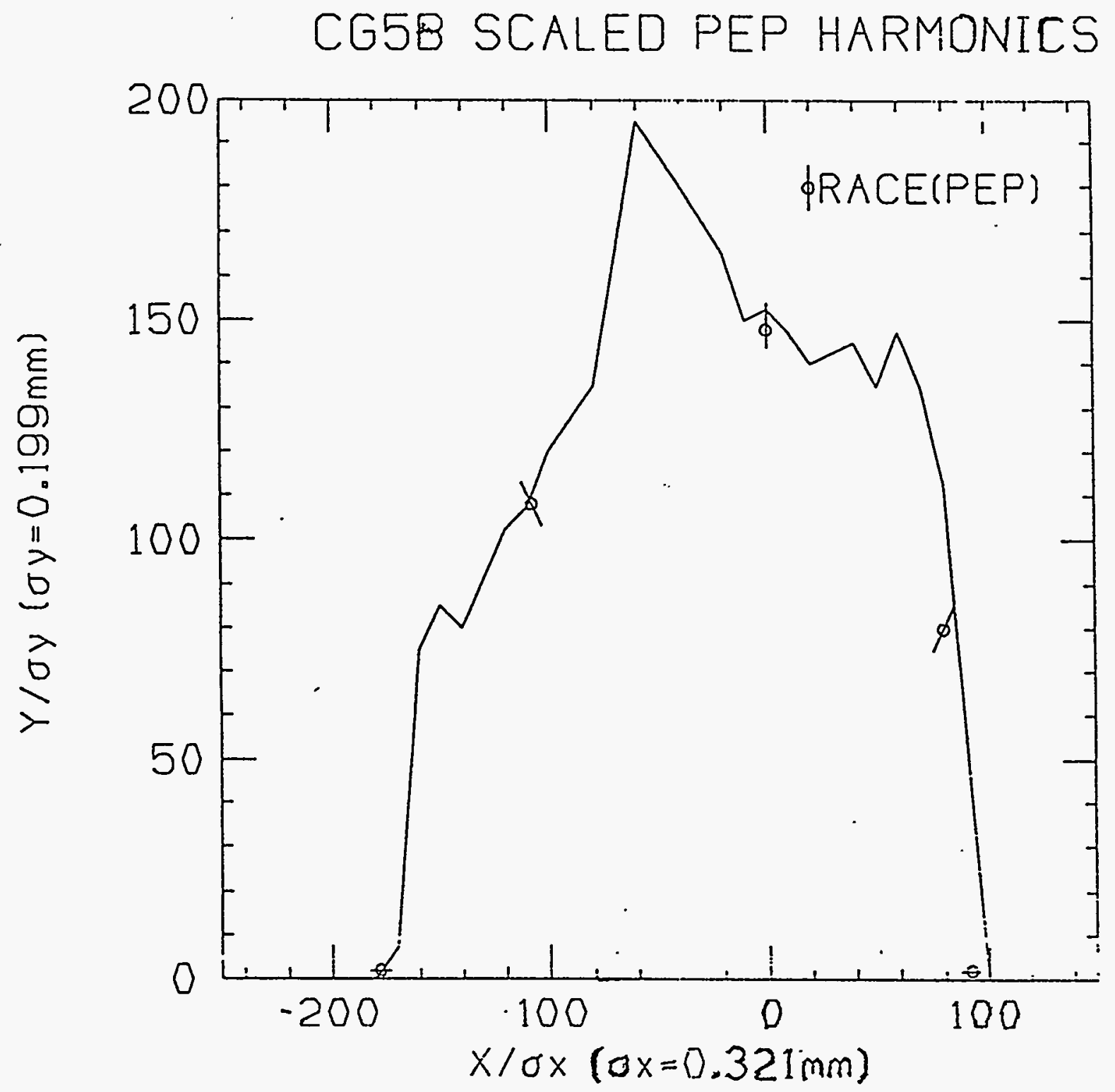

Figure 1

Dynamic aperture for the APS lattice with scaled PEP harmonic coefficients for the quadrupole magnets. The data points and error jbars represent the average and rms spread of the dynamic aperture for 11 random sequences of harmonic coefficients. The solid line represents the dynamic aperture for the ideal lattice and the dashed line the dynamic aperture with a $0.1-m m$ rms quadrupole alignment tolerance. 


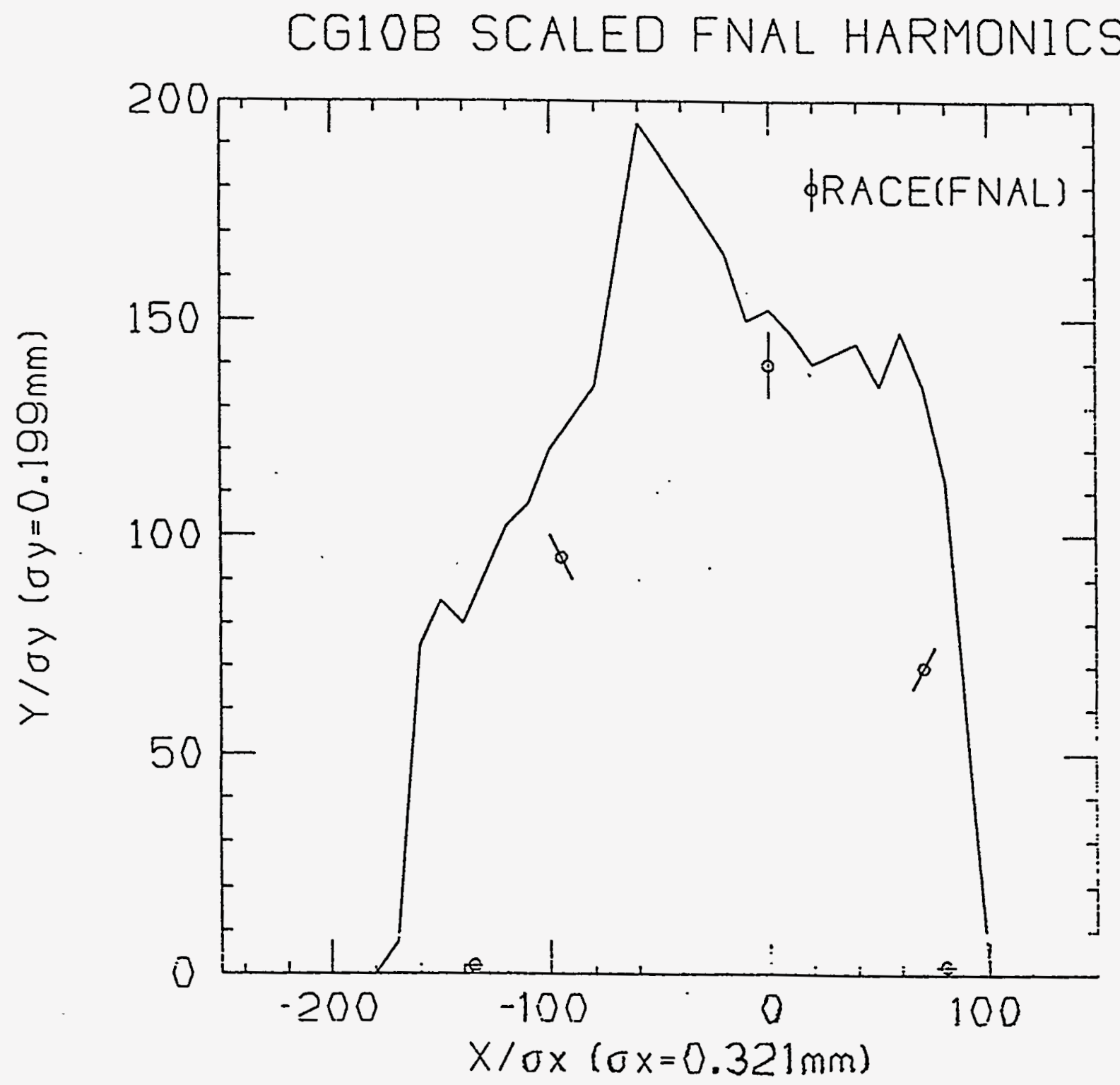

Figure 2

Dynamic aperture for the APS lattice with scaled FNAL harmonic coefficients for the quadrupole magnets. The data points and error jbars represent the average and rms spread of the dynamic aperture for 11 random sequences of harmonic coefficients. The solid line represents the dynamic aperture for the ideal lattice. 


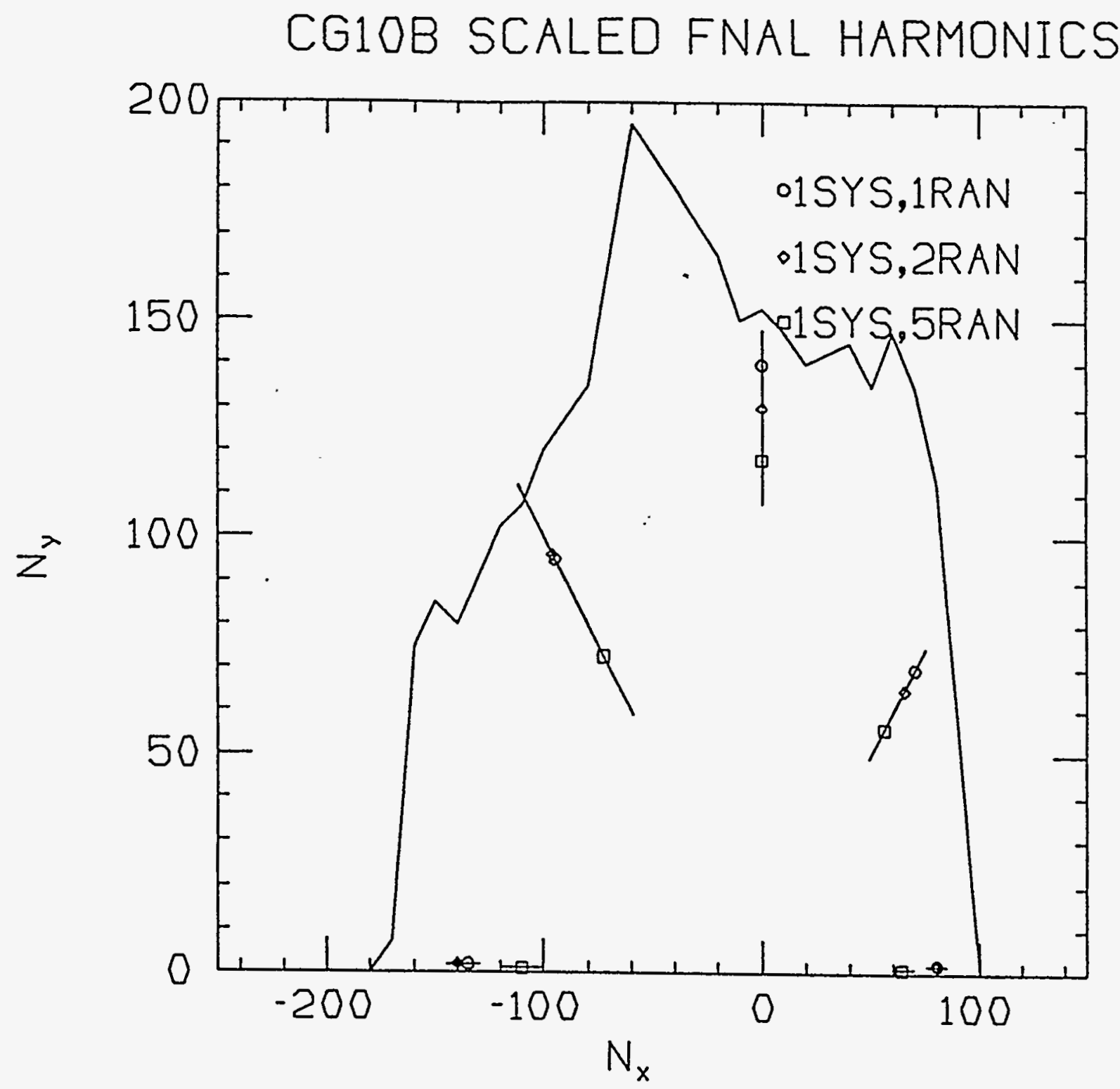

Figure 3

Dynamic aperture for the APS lattice with scaled FNAL harmonic coefficlents for the quadrupole magnets. The circles represent the FNAL coefficients as measured, the diamonds have the random coefficients increased by a factor of two, and the squares have the random coefficients increased by a factor of five. 


\section{CGIOB QP3 AND FNAL RANDOM}

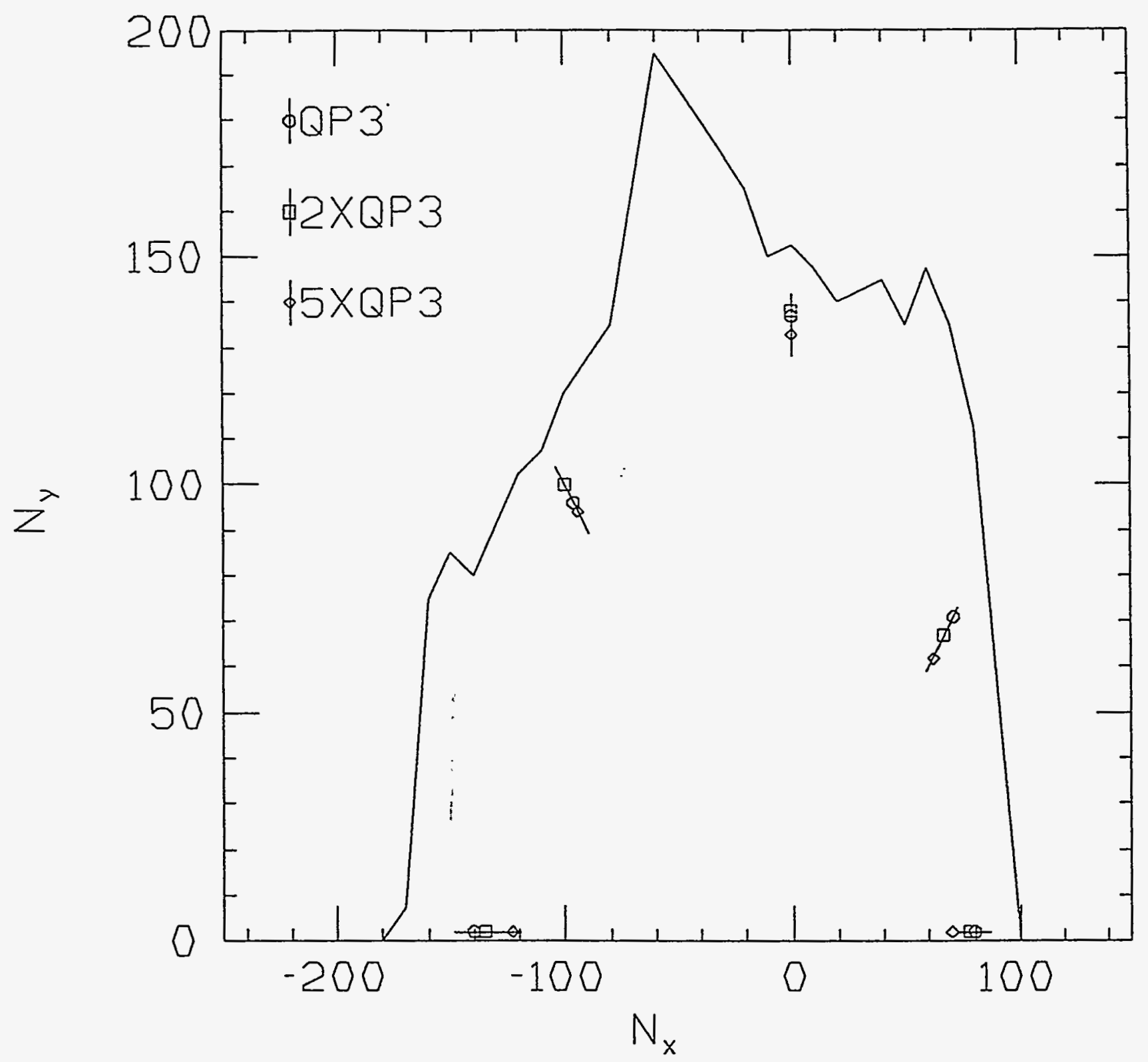

Figure 4

Dynamic aperture for APS lattice with multipole coefficients in the quadrupole given by: $\bar{b}_{n}$ from the QP3 quadrupole design calculations and $\delta b_{n}$ from the scaled FNAL measurements. The circles, squares, and diamonds represent one, two, and five times the QP3 calculated coefficients, respectively. 